\title{
The Color Flavor Locked Phase in the Chromodielectric Model and Quark Stars
}

\author{
L. P. Linares ${ }^{1}$, M. Malheiro ${ }^{1,2}$, \\ 1 Instituto de Física, Universidade Federal Fluminense, Niterói 24210-340, RJ, Brazil \\ ${ }^{2}$ Dep. de Física, Instituto Tecnológico de Aeronáutica, CTA, 12.228-900, São José dos Campos, Brazil
}

A. R. Taurines,

Department of Physics, University of Wales Swansea, Singleton Park, Swansea, SA2 8PP, United Kingdom

and M. Fiolhais

Departamento de Física and Centro de Física Computacional,

Universidade de Coimbra, P-3004-516 Coimbra, Portugal

\section{Received on 18 March, 2006}

\begin{abstract}
Recent results obtained in the Chromodielectric Model (CDM) have shown that strange quark matter at very high densities may appear in two phases, namely a chiral broken and a chiral symmetric phase, which may not be absolutely stable. In the chiral symmetric phase, the abundance of the quarks flavors $u, d$ and $s$ is the same and there are no electrons. In this paper we study an extended version of the Chromodielectric model $(\mathrm{CDM})$ with a BCS quark pairing implemented, and analyze the superconducting color flavor locked phase. We show that the inclusion in the free energy density of a negative term of the diquark condensate guarantees the stability of quark matter. We also analyze the phase transition between matter described by different equations of state and only find a first order transition, at a very low pressure, from the CFL phase to the unpaired strange quark matter, which opens the possibility for a quark-hadron phase transition. Our study has implications in astrophysics, in particular regarding the formation and the structure of compact quark stars. We explicitly show that CFL stars can be absolutely stable and more compact than strange stars.
\end{abstract}

Keywords: Chromodielectric Model; Superconducting color flavor locked phase; CFL stars

\section{INTRODUCTION}

Twenty years ago, Bailin and Love $[1,2]$ conjectured that the attractive channel of the one gluon exchange of QCD could lead to quark pairs of equal momenta and opposite directions near the Fermi surface, in analogy with the electron pairs of the BCS theory [3] of superconductivity in solid state physics. For these pairs to be created (BCS ground state), an attractive interaction, even very small, is enough. The QCD attractive interaction of quarks close to the Fermi surface may lead to the formation of Cooper pairs which condense since they are bosons. The condensates generate gaps in the fermionic spectra which can be understood as the masses of these quasi-quarks. Since quark pairs in QCD cannot be color singlets, the color symmetry must be broken and this phenomenon is known as color superconductivity. In the case of three flavors and massless quarks (including the strange quark), a new phenomenon occurs when a condensate of quark pairs is formed: since the Cooper pairs cannot be flavor isosinglets, both the chiral symmetry and the color symmetry are broken. The condensate formed through the attractive channel of the one gluon exchange exhibits what it is known in literature as Color Flavour Locking (CFL) [4]. The rotation transformations in the flavor space $\mathrm{SU}(3)_{\mathrm{L}}$ are "locked" with the color rotations $\mathrm{SU}(3)_{\text {color }}$, in the sense that the condensate is not invariant for none of these transformations separately, but it is invariant for the simultaneous transformations of the color-flavor $\mathrm{SU}(3)_{\mathrm{L}+\text { color }}$ (the same happens for the condensates formed by the right hand helicity spinores which are invariant under $\mathrm{SU}(3)_{\mathrm{R}+\text { color }}$ rotations). Thus, the chiral sym- metry is broken in the CFL phase due to this new phenomenon and not because of the formation of any quark-antiquark condensate which, at these densities, is simply absent. In this work we are going to investigate, using the chromodieletric model (CDM) [5-7], high density strange quark matter in the CFL phase. We choose this model, where quarks interact with effective mesons fields, since its chiral symmetry is spontaneously broken, generating dynamical masses for quarks (a common feature with NJL models), and since it confines at low energies. The confinement results from an effective field, $\chi$, that can be regarded as an integrated gluon field [8]. At low energies the model yields a good account of the nucleon phenomenology $[9,10]$. Therefore, it is tempting to investigated in the same model whether the confinement information still remains at high densities where we expect the CFL quark matter to be formed.

Moreover, in the framework of the same model, a study of the unpaired three flavor quark matter in $\beta$ equilibrium has been carried out on $[11,12]$ and problems with the stability of this strange matter at high densities have been found [13]. These problems are solved through the introduction of quark pairing, since the strange matter in the superconducting phase has a lower energy per particle than in the unpaired quark phase. Preliminary results have already been presented in $[14,15]$ and, in this work, we will add more results and also analyze the importance of the CFL phase as far as the structure of compact stars is concerned [16].

The CDM model will be used with a quartic potential. This version of the model yields two self-consistent equations of state (EOS) for strange quark matter in $\beta$ equilibrium $[11,12]$. One of them, for low densities, shows up a chiral symmetry 
breaking, a symmetry that is restored in the other one, for high densities. In this one, the Fermi momenta are the same for all three flavors and, therefore, there are no electrons in the neutral quark matter. Similarly, quark matter in the CFL phase also does not contain any electrons, but in the unpaired phase the gap energy is zero $[11,12]$.

We investigated the phase transition between the equations of state and concluded that, at intermediate densities, the color superconducting phase may undergo a transition to the strange unpaired quark matter, if pressure is low enough. However, at sufficiently high densities the transition does not occur. Hence, one should conclude that the CFL state is the true ground state of strange quark matter in the CDM model in that regime.

The superconducting color phase of quark matter may have interesting consequences in astrophysics. Inside a neutron star, densities ten times larger than the nuclear matter density can be reached, with chemical potentials of the order $\mu \sim 400$ to $500 \mathrm{MeV}$. This exceeds the strange quark mass and, for that density, the CFL phase is favored. The existence of a core in a neutron star made up of strange matter, or even the possibility that stars made up entirely of quark matter may exist, was also a motivation for our study. In the normal unpaired quark matter, it was shown that very small compact stars with radius less than $8 \mathrm{~km}$ are metastable $[11,12]$. We will show by means of $M \times R$ diagrams obtained for CFL stars (pure quark stars with the quark matter in the superconducting phase), that it is possible to obtain well compact stars, with maximum mass $M / M_{\odot}$ $=1.43$ and radius $R \sim 7.5 \mathrm{~km}$, where the quark matter is stable $(\varepsilon<\rho M)$. This result seems to indicate that pure quark stars, if they really exist in nature, must be in the CFL phase.

\section{THE CFL PHASE IN THE CHROMODIELECTRIC MODEL}

The Lagrangian density of the CDM can be written as [5-7]

$$
\begin{gathered}
\mathcal{L}=\mathrm{i} \bar{\psi} \gamma^{\mu} \partial_{\mu} \psi+\frac{1}{2}\left(\partial_{\mu} \sigma \partial^{\mu} \sigma+\partial_{\mu} \vec{\pi} \cdot \partial^{\mu} \vec{\pi}\right)-W(\sigma, \vec{\pi})+ \\
+\frac{g}{\chi} \bar{\psi}\left(\sigma+\mathrm{i} \vec{\tau} \cdot \vec{\pi} \gamma_{5}\right) \psi+\frac{g_{s}}{\chi} \bar{\psi}_{s} \psi_{s}+\frac{1}{2} \partial_{\mu} \chi \partial^{\mu} \chi-U(\chi) .
\end{gathered}
$$

The first and second terms describe the quark and meson kinetic energies, respectively, and the third one the chiral meson self-interaction (Mexican hat potential for the scalar $\sigma$ and the pseudoscalar $\pi$ mesons):

$$
W(\sigma, \vec{\pi})=\frac{m_{\sigma}^{2}}{8 f_{\pi}^{2}}\left(\sigma^{2}+\pi^{2}-f_{\pi}^{2}\right)^{2},
$$

where $f_{\pi}$ is the pion decay constant.

The fourth and fifth terms in (1) describe the meson-quark interaction: the former refers only to the two light quark flavors $(u$ and $d)$ and the latter to the strange quark $s$, so the model is extended to the strange sector. The last two terms in the Lagrangian density (1) refer to the dynamical confining $\chi$ field, namely to its kinetic (sixth term) and potential, $U(\chi)$, energies. We use a CDM version with the following quartic potential for the $\chi$ field:

$$
\begin{gathered}
U(\chi)=\frac{1}{2} m_{\chi}^{2} \chi^{2} \\
\times\left[1+\left(\frac{8 \eta^{4}}{\gamma^{2}}-2\right)\left(\frac{\chi}{\gamma m_{\chi}}\right)+\left(1-\frac{6 \eta^{4}}{\gamma^{2}}\right)\left(\frac{\chi}{\gamma m_{\chi}}\right)^{2}\right]
\end{gathered}
$$

where $m_{\chi}$ is the mass of the $\chi$ field. The potential has an absolute minimum at $\chi=0$. The other two constants acquire a simple meaning, when the potential is written as in Eq. (3): $\gamma$ defines the position of the second (local) minimum, located at $\chi=\gamma m_{\chi}$, and $\eta$ the value of the potential at this minimum: $U\left(\gamma m_{\chi}\right)=\left(\eta m_{\chi}\right)^{4}$. The range for $\eta$ in this work is the same as in $[11,13]$.

In the mean field approximation, where the meson fields are constant classical fields, the energy density for an homogeneous system of $u, d$ and $s$ quarks, interacting with the $\chi$ and $\sigma$ fields is given by [11-13]:

$$
\begin{aligned}
\varepsilon= & \alpha \sum_{i=u, d, s} \int_{0}^{k_{i}} \frac{d^{3} k}{(2 \pi)^{3}} \sqrt{k^{2}+m_{i}(\sigma, \chi)^{2}}+U(\chi) \\
& +\frac{m_{\sigma}^{2}}{8 f_{\pi}^{2}}\left(\sigma^{2}-f_{\pi}^{2}\right)^{2}
\end{aligned}
$$

(due to parity, the terms involving the pion field vanish in this approximation). The degeneracy factor is $\alpha=2(\operatorname{spin}) \times 3$ (color) $=6$, and $f_{\pi}=93 \mathrm{MeV}$ and $m_{\sigma}=1.2 \mathrm{GeV}$. Since we are dealing with an infinite and homogeneous system, the fermions are described by plain waves. The last two terms correspond to the $\chi$ and $\sigma$ potential energies, respectively. The first term of Eq. (4) is the relativistic kinetic energy of a fermion gas, corresponding to the three quark flavors.

The Fermi momentum of each quark, $k_{i}$, is related to the corresponding density, $\rho_{i}$, through:

$$
\rho_{i}=\alpha \frac{k_{i}^{3}}{6 \pi^{2}}=\frac{k_{i}^{3}}{\pi^{2}}
$$

where $i=u, d$ and $s$.

The meson-quark interaction terms generate dynamical quark masses that are all different [18], namely

$$
m_{u}(\sigma, \chi)=\frac{g_{u} \sigma}{\chi f_{\pi}}, m_{d}(\sigma, \chi)=\frac{g_{d} \sigma}{\chi f_{\pi}}, m_{s}(\chi)=\frac{g_{s}}{\chi},
$$

and the coupling constants for the $u, d$ and $s$ quarks are given, respectively, by

$$
g_{u}=g\left(f_{\pi}+\xi_{3}\right), g_{d}=g\left(f_{\pi}-\xi_{3}\right), g_{s}=g\left(2 f_{K}-f_{\pi}\right)
$$

where $\xi_{3}=-0.75 \mathrm{MeV}$ and the kaon decay constant $f_{K}=113$ $\mathrm{MeV}$. The parameters that better reproduce the nucleon properties in this model are the coupling constant, $g=0.023 \mathrm{GeV}$, the $\chi$ field mass, $m_{\chi}=1.7 \mathrm{GeV}$ and $\gamma=0.2$, which we keep fixed at this value as in $[11,17]$. Finally, since we are interested in the study of strange quark matter in compact stars, we have to take in account the $\beta$ equilibrium. Thus a relativistic free electron gas should be added [11, 13] to the energy density, Eq. (4). 
We now extend the chromodieletric model presented so far in order to include the diquark condensate. The superconducting CFL phase appears when an attractive term responsible for the quark pairing is introduced. This term reads $-3\left(\frac{\Delta \mu}{\pi}\right)^{2}$ and its effect is to decrease the free energy of the system. The gap energy, $\Delta$, in principle, depends on the quark chemical potential, $\mu$, in the range of neutron star densities ( $\mu$ between 400 to $500 \mathrm{MeV}$ ), but we are going to take it as a constant [19]. This new term has not been derived in the CDM but rather in the framework of QCD, in the one gluon approximation. That interaction can be modelled by a four fermion interaction that is linearized, in the spirit of NJL models [19, 20]. In the CDM, a similar interaction could be generated through the introduction of an effective meson field that would represent the diquark pair. In this case, it would be possible to derive a self-consistent equation for the gap as a function of the density. Such a study is going to be carried on in a future work, but here, we will be working in the most simple BCS approximation, assuming the gap energy to be constant.

Moreover, as noted in [11, 12], the CDM model at high density predicts an EOS for the unpaired strange quark matter that is essentially the same as the one obtained by a perturbative QCD expansion [21]. Since, the CDM model incorporates the main QCD dynamics at high densities, we would expect this to be also the case for the diquark condensate, making it reasonable the introduction of the QCD pairing term in the CDM.

For systems at zero temperature, the grand-potential divided by the system volume is given by

$$
\Omega=\varepsilon-\mu \rho
$$

where $\varepsilon$ is the energy density, $\mu$ is the chemical potential and $\rho$ the particle density.

The grand-potential density of CDM in the superconducting color phase (CFL), is given by $[14,15]$

$$
\begin{aligned}
\Omega_{\mathrm{CFL}}= & \alpha \sum_{i=u, d, s} \int_{0}^{k_{i}} \frac{d^{3} k}{(2 \pi)^{3}}\left(\sqrt{k^{2}+m_{i}^{2}}-\mu_{i}\right) \\
& -3\left(\frac{\Delta \mu}{\pi}\right)^{2}+U(\chi)+W(\sigma, \vec{\pi})
\end{aligned}
$$

where the quark masses $m_{i}$ are given by Eqs. (6), and the meson potentials by Eqs. (2) and (3). Using spherical symmetry and $\alpha=6$, Eq. (9) can be written as:

$$
\Omega_{\mathrm{CFL}}=\varepsilon_{k}-\frac{1}{\pi^{2}} \sum_{i=u, d, s} \mu_{i} k_{i}^{3}-3\left(\frac{\Delta \mu}{\pi}\right)^{2}+U(\chi)+W(\sigma, \vec{\pi})
$$

where

$$
\varepsilon_{k}=\frac{3}{\pi^{2}} \sum_{i=u, d, s} \int_{0}^{k_{i}}\left(k^{2} \sqrt{\left(k^{2}+m_{i}^{2}\right)}\right) d k
$$

is the kinetic energy for a relativistic quark gas [14, 15, 22].

Inserting (10) into (8), we obtain the following quark matter energy density:

$$
\begin{aligned}
\varepsilon= & \varepsilon_{k}-\frac{1}{\pi^{2}} \sum_{i=u, d, s} \mu_{i} k_{i}^{3}-3\left(\frac{\Delta \mu}{\pi}\right)^{2}+\sum_{i=u, d, s} \mu_{i} \rho_{i} \\
& +U(\chi)+W(\sigma, \vec{\pi})
\end{aligned}
$$

In the CFL phase, in order to guarantee the local charge neutrality [22], the Fermi momenta $\left(k_{i}=\sqrt{\mu_{i}^{2}-m_{i}^{2}}\right)$ of all quarks are equal, as explained previously:

$$
k_{f}=k_{u}=k_{d}=k_{s}=2 \mu-\sqrt{\mu^{2}+\frac{m_{s}^{2}}{3}}
$$

where we define the quark chemical potential $\mu$ by

$$
3 \mu=\sum_{i=u, d, s} \mu_{i}
$$

and admit that the $u$ and $d$ quark masses are very small [22].

When we derive the grand-potential, given by Eq. (10), to respect the chemical potential of each quark, $\mu_{i}$, we obtain the same quark density for $u, d$ and $s$ if we take in account, as explained before, that all quarks have the same Fermi momentum in the CFL phase. Thus, if we use the definition of the average quark chemical potential, $\mu$, expressed by Eq. (14), the baryonic density $\rho$ is given by

$$
\rho=\rho_{u}=\rho_{d}=\rho_{s}=\frac{1}{\pi^{2}}\left(k_{f}^{3}+2 \Delta^{2} \mu\right) .
$$

As we can see from this expression, the baryonic density in the superconducting phase also depends on the value of the pairing constant, $\Delta$, and not only on the Fermi momentum, $k_{f}$. This result reflects the well known fact that BCS theory violates the particle number conservation since now there is a dependence on the number of diquark pairs. Thus, for a given density, the larger the gap, $\Delta$, the smaller the Fermi momenta. As we will see, this will affect the contribution to the energy density of the quark kinetic energy term given in Eq. (12).

Inserting (13), (14) and (15) back into Eq. (12), one gets

$$
\begin{aligned}
\varepsilon= & \varepsilon_{k}-\frac{3 \mu k_{f}^{3}}{\pi^{2}}-3\left(\frac{\Delta \mu}{\pi}\right)^{2}+U(\chi)+W(\sigma, \pi) \\
& +\frac{3 \mu}{\pi^{2}}\left(k_{f}^{3}+2 \Delta^{2} \mu\right) .
\end{aligned}
$$

This expression can still be re-arranged so that the energy density of the CFL phase of strange quark matter can be cast in the form:

$$
\varepsilon=\varepsilon_{k}+3\left(\frac{\Delta \mu}{\pi}\right)^{2}+U(\chi)+W(\sigma, \pi),
$$

where $\varepsilon_{k}$ is given by Eq. (11).

\section{RESULTS}

As for the strange quark matter without pairing $[11,12]$, the model accommodates two solutions: one for small values of $\chi$ 
(solution I) and the other one for large values of this field, near to the second minimum of the confining potential $\chi \sim \gamma m_{\chi}$ (solution II). Keeping the masses of the mesons and of the $\chi$ field as fixed parameters, the free parameters of the model are the coupling constant $g$, and the $\gamma$ and $\eta$ parameters of the $\chi$ potential energy. These two parameters matter for solution II since they determine the second minimum $\mathrm{U}(\chi)$ potential (the other minimum is always at $\chi=0$ ). However, the sensitivity of the EOS for solution II on the parameter $\gamma$ is negligible, since all three quark masses are very small because they are determined by the inverse of the $\chi$ field, which is always large for solution II [11]. On the other hand, since the dependence of both EOS on the coupling constant $g$ is only through the quark dynamical masses, which is almost zero in solution II, the EOS for this solution only depends on the parameter $\eta$ which fixes the value of the potential energy at the local minimum. Moreover, for both solutions, the self-consistent scalar field is $\sigma \sim f_{\pi}$, so the contribution of the Mexican hat potential almost vanishes. The dependence of solution II on just one parameter explains why the results for this EOS in the superconducting phase are similar to those obtained using the MIT bag model [22]. Increasing $\eta$, which means increasing the potential energy at the local minimum, is equivalent to increase the bag constant, $B$, of the MIT bag model. So, as in [22], the CFL strange quark matter of the solution II is more stable than the unpaired one.

In previous works, the stability of the unpaired strange quark matter in $\beta$ equilibrium [11] for solution II $(\varepsilon<\rho M)$ was studied as a function of the model parameters. It was shown that, for large confining potential energies (large values of $\eta$ ), the quark matter was unstable. However, as it can seen from Eq. (10), the presence of the attractive pairing term in the grand-potential may balance the positive contribution coming from the confining potential energy, so the supercondutivity of quark matter turns out to be the way to solve the meta-stabilidade of the unpaired quark matter. From Eqs. (8) and (17), and taking into account that the grand-potential vanishes when the energy per particle has a minimum (zero pressure, $\mu=\varepsilon / \rho$ ), one readily obtains the relationship between the maximum value of $\eta$ and the gap constant $\Delta$, for which the CFL quark matter in the solution II (where the quarks are almost massless) is still stable $(\varepsilon / \rho=M)$ :

$$
\begin{aligned}
\eta_{\max } & \sim \frac{M}{m_{\chi}}\left(\frac{1}{108 \pi^{2}}\right)^{1 / 4}\left(1+\frac{6 \Delta^{2}}{M}\right)^{1 / 4} \\
& \sim 0.0966\left(1+\frac{6 \Delta^{2}}{M}\right)^{1 / 4} .
\end{aligned}
$$

This expression shows that, for unpaired quark matter $(\Delta=0)$, $\eta>0.0966$ will make the quark matter unstable, corroborating what was obtained in Ref. [11]. For the CFL phase with a strong pairing interaction, large values of $\eta$ are still allowed (larger potential energy) by the stability condition (18).

In Fig. 1-a, the energy per particle for the solution II is shown as a function of the baryonic density, for various pairing parameters. As we can observe, increasing of the pairing interaction $\Delta$ lowers the energy per particle, leading to a more robust quark matter stability. In the case of solution I $(U(\chi) \sim 0)$, the strange matter is stable only for values of

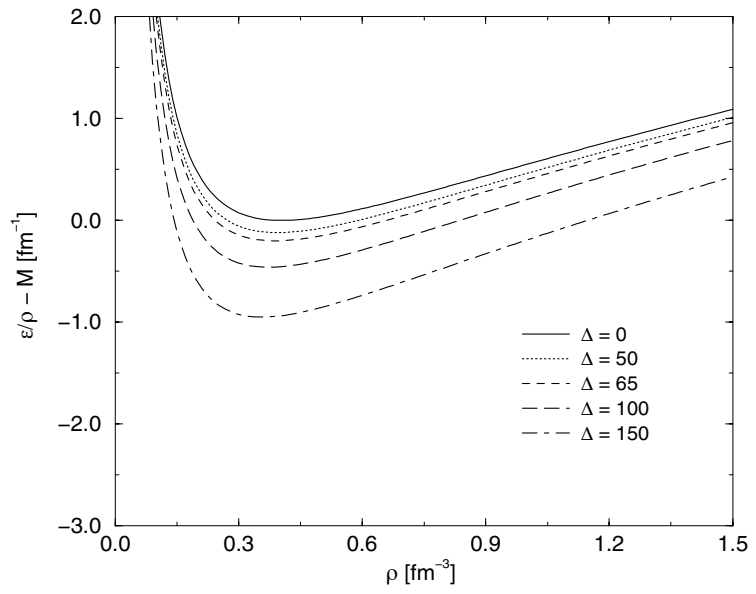

(a)

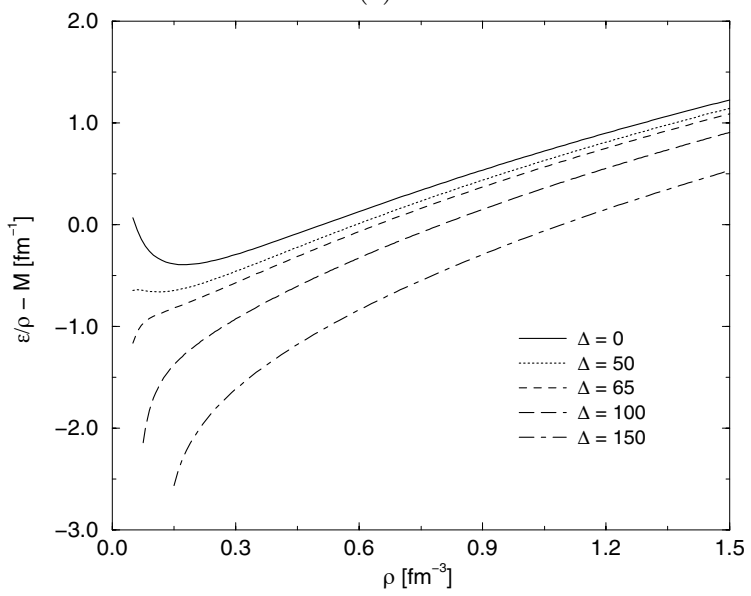

(b)

FIG. 1: Energy per particle as a function of the baryonic density with $\eta=0.0966$ and different $\Delta$ pairing interaction for the solution II (a) and for solution I (b).

$\Delta \lesssim 65 \mathrm{MeV}$. This happens because only for these values of $\Delta$, the pressure vanishes producing a minimum in Fig. 1-b. For larger values of the pairing, the energy per particle does not show a minimum, and the matter collapses for low densities. In solution I, the values for the $\chi$ field are very small and the dynamical quark masses large. This collapse indicates that the superconducting phase of massive quarks is not favored.

After analyzing the effect of the strong pairing in the energy density, it is worth investigating its effect on the pressure for the two solutions. First, let us take $\eta=0.0966$ and increase the other parameter, starting at $\Delta=0$. In Fig. 2-a we present the EOS for solution II, while the EOS for solution I is shown in Fig. 2-b. In these two figures we can observe that, for a given energy density, increasing the pairing strength leads to a corresponding increase in the pressure. This result shows that the pairing interaction makes the EOS more stiff in the CFL phase.

In order to study the possible phase transitions between different EOS (paired and unpaired strange quark matter) we present, in Fig. 3 a plot of the pressure versus the quark chemical potential, $\mu$. These curves indicate a phase transi- 


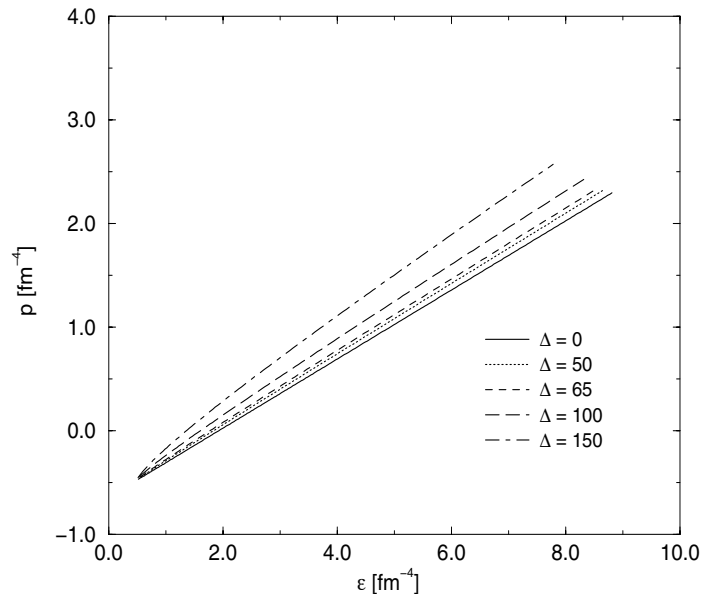

(a)

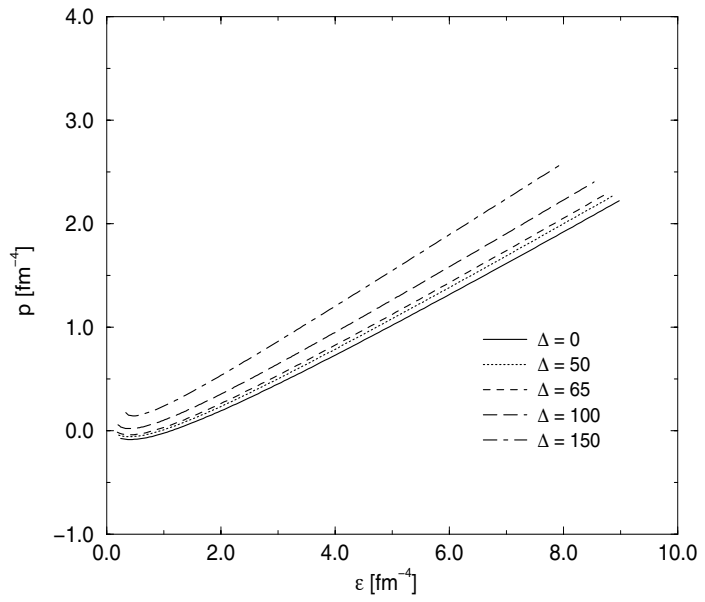

(b)

FIG. 2: Equation of state for different pairing parameters for the solution I (a) and solution II (b).

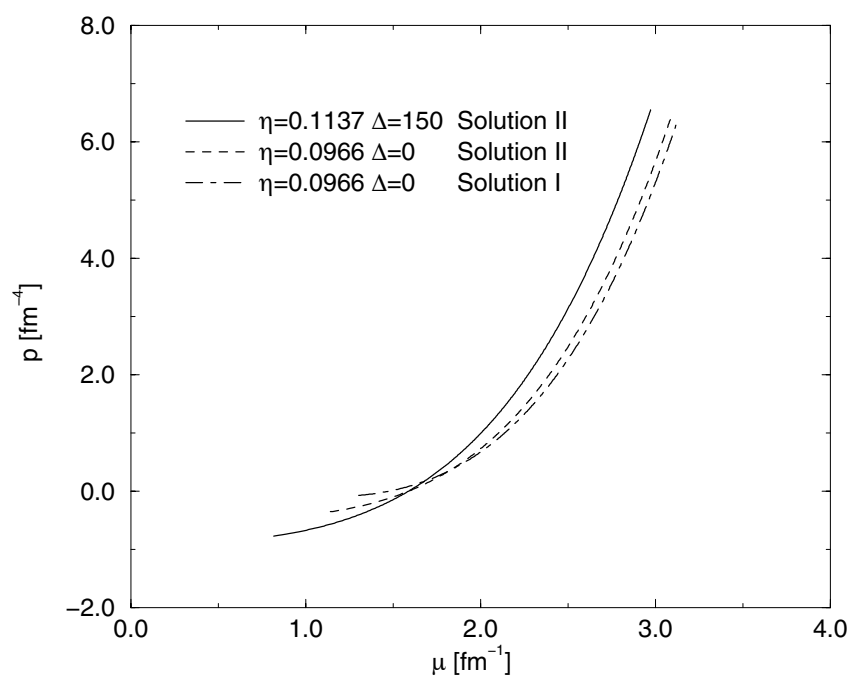

FIG. 3: Pressure as a function of the quark chemical potential for different quark EOS as explained in the text. tion from the CFL quark matter (solution II) to the unpaired quark matter in $\beta$ equilibrium (solution I). We remind that the unpaired strange matter appears in the chiral broken phase with massive quarks [11]. This is a first order phase transition, indicating the existence of a mixing phase between these two phases. The system changes from the CFL phase, with a strong pairing interaction $(\Delta=150 \mathrm{MeV})$, at a density around $0.67 \mathrm{fm}^{-3} \sim 4.5 \rho_{0}$ to a smaller density of around $0.41 \mathrm{fm}^{-3} \sim 3.0 \rho_{0}\left(\rho_{0}=0.15 \mathrm{fm}^{-3}\right.$ is the normal nuclear matter density), with the quark matter in the unpaired phase and in $\beta$ equilibrium. This result shows that, in the CDM model, there is a parameter range for which the CFL phase coexists with the chiral broken phase of unpaired strange quark matter. Since this transition occurs at a much too low pressure, even close to zero, it is possible that a quark-hadron transition also takes place. To investigate this possibility some hadronic EOS would be required. This is certainly a topic for future work; here we rather concentrated on the strange quark matter transitions.

One motivation for the present work, already pointed out in the Introduction, is to investigate whether bare quark stars in the CFL phase are absolutely stable, and how compact they are in comparison with strange stars made up of unpaired quark matter in $\beta$ equilibrium. To address this issue, we solved the Oppenheimer-Volkov equation for relativistic stars and obtained the mass $v s$. radius diagram for the bare quark stars, shown in Fig. 4. As the results show, keeping the pairing interaction fixed and increasing the potential energy (i.e. increasing $\eta$ ) we get CFL stars that are very compact and still stable in contrast with the strange stars made up of unpaired quark matter, which are metastable [11].

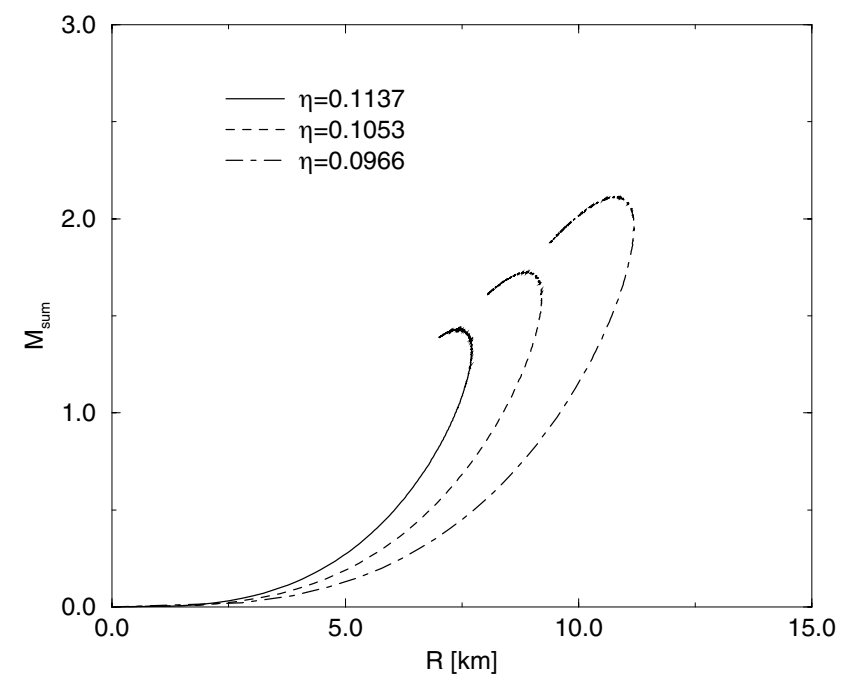

FIG. 4: Mass - radius diagram with $\Delta=150 \mathrm{MeV}$ and different values of $\eta$.

We present in Table I the results for the maximum mass, $M$ of CFL strange stars (in units of the solar mass, $M_{\odot}$ ), the radius, $R$, and the central energy density, $\varepsilon_{\mathrm{c}}$, for different values of $\eta$ (all yielding a stable quark matter). As we can read from this table, relatively large maximum masses for CFL stars can 


\begin{tabular}{|c|c||c|c|c|}
\hline$\eta$ & $\Delta[\mathrm{MeV}]$ & $M\left[M_{\odot}\right]$ & $R[\mathrm{~km}]$ & $\varepsilon_{\mathrm{c}}\left[\mathrm{fm}^{-4}\right]$ \\
\hline 0.0966 & 0 & 1.56 & 8.48 & 9.62 \\
\cline { 2 - 5 } & 100 & 1.81 & 9.61 & 7.18 \\
\cline { 2 - 5 } & 150 & 2.11 & 10.81 & 5.72 \\
\hline 0.1053 & 100 & 1.49 & 7.94 & 10.62 \\
\cline { 2 - 5 } & 150 & 1.72 & 8.89 & 8.46 \\
\hline 0.1137 & 150 & 1.43 & 7.45 & 11.87 \\
\hline
\end{tabular}

TABLE I: Values of the maximum mass $M$, radius $R$ and central density energy $\varepsilon_{\mathrm{c}}$ for the stable CFL star with different values of $\eta$ and $\Delta$.

be obtained. Most of the of neutrons stars have a mass $M \sim$ $1.4 M_{\odot}$. It is interesting that in the maximum limit of quark matter stability (large $\eta$, for $\Delta=150 \mathrm{MeV}$ ) the maximum mass is around this value but with a much smaller radius only $R \leq 8 \mathrm{~km}-$ in comparison with the radius range from 10 to $14 \mathrm{~km}$ of a typical neutron star.

With the parameters considered in our model, the maximum limit for the potential energy and for the gap energy to have stable quark matter are $\eta=0.1137$ and $\Delta=150 \mathrm{MeV}$ respectively. In this case we get very compact CFL stars as shown in Fig. 4, which are stable, something that is not possible to obtain in the case of the unpaired quark matter without facing the problem of the metastability [11]. Thus, we can conclude that the color superconducting matter is stable and CFL stars can be rather small.

\section{CONCLUSIONS}

Through the introduction of a QCD inspired diquark interaction in the CDM model, we have studied the superconducting color flavor locked phase of strange quark matter at high densities, and compare with the previous results obtained in this model for the unpaired quark matter in $\beta$ equilibrium. Our findings can be summarized as follow:

1. The CFL quark matter is more stable that the unpaired phase; when pairing is allowed, the range of the $\eta$ parameter (which mimics the bag constant) is wider;

2. For standard CDM parameters, the CFL phase is absolutely stable at high densities, showing a first order phase transition to the $\beta$ equilibrium unpaired quark phase at very small pressures;

3. CFL stars are stable and can be very compact with a mass similar to a neutron star mass but a smaller radius of the order of $8 \mathrm{~km}$.

\section{Acknowledgments}

This work was supported by Capes and CNPq, Brazil, by FCT (POCTI/FEDER program), Portugal, and by CNPq/GRICES Brazilian-Portuguese scientific exchange program.
[1] D. Bailin and A. Love, Phys. Rept. 107, 325 (1984)

[2] D. Bailin and A. Love, Supersymmetric Gauge Field Theory and String Theory, (IOP, London, 1994).

[3] J. Bardeen, L. N. Cooper, and J. R. Schrieffer, Phys. Rev. 106, 162 (1957); 108, 1175 (1957).

[4] M. Alford, K. Rajagopal, and F. Wilczek, Nucl. Phys. B 537, 433 (1999)

[5] M. K. Banerjee, Prog. Part. Nucl. Phys. 31, 77 (1993).

[6] M. C. Birse, Prog. Part. Nucl. Phys. 25, 1 (1990).

[7] H. J. Pirner, Prog. Part. Nucl. Phys. 29, 33 (1992).

[8] H. B. Nielsen and A. Patkós, Nucl. Phys. B 195, 137 (1982).

[9] T. Neuber, M. Fiolhais, K. Goeke, and J. N. Urbano, Nucl. Phys. A 560, 909 (1993).

[10] A. Drago, M. Fiolhais, and U. Tambini, Nucl. Phys. A 609, 488 (1996).

[11] M. Malheiro, M. Fiolhais, and A. R. Taurines, J. Phys. G 29, 1045 (2003).

[12] M. Malheiro, E. O. Azevedo, L. G. Nuss, M. Fiolhais, and A. R. Taurines, AIP Conference Proceedings 631, (1) 658 (2002), hep-ph/0111148

[13] M. Fiolhais, M. Malheiro, and A. R. Taurines, Stability of Quark Matter and Quark Stars, Proceedings of the 4th international conference "New Worlds in Astroparticle Physics", Faro 2002, A. Krasnitz et al. (Eds.), World Scientific, Singapore, 123
(2003).

[14] M. Fiolhais, L. P. Linares, M. Malheiro, and A. Taurines, Bled Workshops in Physics, 5, 18 (2004).

[15] L. P. Linares, M. Malheiro, M. Fiolhais, and A. Taurines, AIP Conference Proceedings 739, 488 (2004).

[16] L. P. Linares Color superconducting phase in the chromodielectric model and applications to quark stars, Thesis (in Portuguese), Universidade Federal Fluminense, Niterói, 2005 (unpublished).

[17] A. Drago, M. Fiolhais, and U. Tambini, Nucl. Phys. A 588, 801 (1995).

[18] J. A. McGovern and M. Birse, Nucl. Phys. A 506, 367 (1990); Nucl. Phys. 506, 392 (1990).

[19] K. Rajagopal and F. Wilczek, The Condensed Matter Physics of $Q C D$, Chapter 35 'At the Frontier of Particle Physics / Handbook of QCD', M. Shifman, ed., (World Scientific), vol. 3 20612151.(2001), hep-ph/0011333

[20] M. Alford, Color Superconducting Quark Matter Ann. Rev. Nucl. Part. Sci. 51, 131-160 (2001), hep-ph/0102047

[21] E. S. Fraga, R. D. Pisarski, and J. Schaffner-Bielich, Phys. Rev. D 63, 121702 (2002).

[22] G. Lugones and J. E. Horvath, Phys. Rev. D 66, 074017 (2002). 